Relations industrielles

Industrial Relations

\title{
Nos collaborateurs
}

Volume 2, numéro 8, avril 1947

URI : https://id.erudit.org/iderudit/1024065ar

DOI : https://doi.org/10.7202/1024065ar

Aller au sommaire du numéro

\section{Éditeur(s)}

Département des relations industrielles de l’Université Laval

ISSN

0034-379X (imprimé)

1703-8138 (numérique)

Découvrir la revue

Citer cet article

(1947). Nos collaborateurs. Relations industrielles / Industrial Relations, 2(8),

3-3. https://doi.org/10.7202/1024065ar

Tous droits réservés (C Département des relations industrielles de l’Université Laval, 1947
Ce document est protégé par la loi sur le droit d'auteur. L'utilisation des services d'Érudit (y compris la reproduction) est assujettie à sa politique d'utilisation que vous pouvez consulter en ligne.

https://apropos.erudit.org/fr/usagers/politique-dutilisation/ 
nier général de la C.T.C.C., ce dernier, au chapitre de l'atelier ferme, précise comme suit la même pensée:

\& Un objectera peut-etre que les unionistes, en agissant ainsi, entravent indirectement la liberté d'embauchage de l'employeur et la liberté de travail des non-unionistes. Il est toujours permis de poser un acte bon ou indifférent de sa nature duquel s'ensuivra deux effets: l'un bon, l'autre mauvais, pourvu que l'on puisse alléguer des raisons d'une gravité proportionnée aux effets que la volonté ne peut empêcher. Les bons effets sont d'ordre professionnel et social. Les mauvais effets sont d ordre particulier ».

Mgr L.-A. Paquet, dont l'autorité, dans les domaines de la théologie, de la philosophie et de la sociologie, n’a jamais été contestée, ni de son vivant, ni depuis sa mort, écrivait de son côté: «Nous ne voulons point condamner les ouvriers syndiqués qui, sans rupture de contrat d'aucune sorte, sans danger pour lordre public ni pour le bien général de leurs familles, dans l'intérêt de l'union dont ils font partie et qu'ils jugent traitée de façon inéquitable, quittent ensemble, délibérément et pacifiquement, l'atelier où ils travaillent. En vertu de la liberté du travail dont jouissent et les individus et les unions, ils ont droit de poser au patron leurs justes conditions, de même que le patron, en acceptant le travail offert, peut poser les siennes 》. (1)

Une association patronale s'est prononcée ouvertement en faveur de l'atelier ferıné, à Washington, devant un comité parlementaire. récemment. On peut lire, en eftet, dans Newsweek, 10 février 1947, (page 67), ce qui suit: «A solid front of employers favoring legislation to outlaw the closed shop was broken last week by Victor S. Riesenfeld, chairman since 1939 of the U. S. Clothing Manufacturers Association's bargaining committee. Closed-shop contracts between clothing manufacturers and the CIO Amalgamated Clothing Workers, said Riesenfeld, have been a stabilising and constructive influence. 》

Dans un court article intitulé «The closed shop and legal authority », la revue anglaise «The Industrial Law Review》, Vol. 1, no. 7, Décembre 1946, cite une décision de la Chambre des Lords, dans la cause de Crofter Harris Tweed Co. Ltd. vs Weitch (1942). A. C. Voici l'extrait de la revue: «Here a trade union in order to prevent the employment of non-unionists being employed in the northwest of Scotland, placed an embargo on the port of Stornaway for Harris Tweed woven from mainland yarn, as opposed to the island yarn made by members of the union concerned. It was decided that this embargo was lawful, Lord Wright said: «The individual's right to freedom in conducting a trade is not absolute or unconditional; it is only a particular aspect of the citizen's right to personal freedom, and like other aspects of that right, is qualified by various legal limitations, either by Statute or by Common Law. Such limitations are inevitable in organized societies, where the rights of individuals may clash. »

Ce qui précède traite de l'atelier fermé en faveur d'un syndicat qui est en face de non-syndiqués. Mais si le Syndicat est en face, non plus de non-syndiqués, mais d'un autre ou de plusieurs autres syndicats ? Il importe de clarifier cette situation. L'atelier fermé vise à empêcher le sabotage des métiers et des salaires par les non-syndiqués, et non à supprimer le pluralisme syndical et à créer le syndicat unique et obligatoire. Il ne peut y avoir qu'un syndicat légitime et capable de défendre les intérêts professionnels des travailleurs. Dans «Catéchisme syndical », M. l'abbé Georges Côté, propose, avec raison, la solution suivante: \&Il y a lieu, dans ce cas, pour ces unions de s'entendre par le moyen du cartel. L'atelier fermé a pour but premier d'empècher les non-unionistes (scabs) de nuire à l'intérêt du métier, non d'éliminer les groupements syndicaux. Conséquemment il serait abusif de la part d'un syndicat que de tenter d'imposer l'atelier fermé contre une union organisée dans la même industrie ou le même métier. Si, d'un autre côté, cette dernière union compromet l'intérêt professionnel dans ses agissements, il est permis de la traiter comme des non-unionistes et d'user de la cessation concertée du travail pour empêcher ce groupement syndical de nuire à l'intérêt du métier. »

La sécurité syndicale, en résumé, est un phénomène social provoqué par les syndicats de travailleurs pour assurer leur sur. vivance. Ces syndicats, du moment qu'ils jouissent de leur pleine liberté, ont droit de choisir parmi les moyens qui leur paraissent les meilleurs pour atteindre leur fin. Et du moment que les moyens choisis sont bons et inattaquables devant la morale sociale, il n'appartient à personne d'intervenir pour leur imposer de choisir parmi les seuls moyens paisibles qui ne dérangent pas trop le patronat, les gouvernements et les non-syndiqués.

Les syndicats de travailleurs doivent être puissants. Leur rôle ne se borne pas seulement aux négociations collectives. Dans

(1) Semaine Sociale du Canada, deuxième session, Québec 1921, p. 46. une société bien organisée, on doit les inviter à participer à l'étude et à la solution de tous les problèmes économiques et sociaux.

En terminant, une dernière citation. Dans son rapport à la Conférence Internationale du Travail de New York (1941), le Directeur du Bureau International du Travail ne craignait pas d'affirmer:

«On sait que les problèmes sociaux et économiques ne peuvent être étudiés et traités dan; l'isolement. Si les objectifs sociaux doivent constituer l'élément fondamental de la politique générale, la participation des organisations d'employeurs et de travailleurs à tous les stades de l'établissement et de l'application de la politique économique et sociale n'est pas seulement désirable; elle est indispensable. »

Gérard PICARD

\section{LE MOUVEMENT OUVRIER CANADIEN}

«Ce volume, d'une vibrante actualité, propose à la connaissance et à la méditation du lecteur le problème du travail organisé et de son action dans les cadres de notre économie et mems audelà ». C'est en ces termes que M. Edouard Montpetit présente louvrage de M. Jean-Pierre Després: Le mouvement ouvrier canadien. (1)

Sauf pour les mitiés, il est très difficile de se débrouiller à travers les différents groupements des travailleurs au Canada. Le volume du jeune professeur de Laval, docteur en sciences sociales et itujourd'hui membre du personnel du Bureau international du Travail à Genève, vient combler un vide dans la littérature canadienne des relations du travail. On y trouve brièvement expliquée la structure du syndicalisme tant sur le plan professionnel que géographique. L'auteur brosse une esquisse historique du mouvement syndical depuis ses premiers développements jusqu'à nos jours; il décrit les principes et les tendances doctrinales de chacun des groupements importants qui se partagent l'adhésion des ouvriers canadiens: le Congrès des Métiers et du Travail, la Confédération des Travailleurs catholiques du Canada et le Congrès canadien du Travail. Enfin, il parle des aspirations politiques de la classe ouvrière et de sa participation à l'action internationale.

En appendice, M. Després touche à différents sujets en rapport avec le syndicalisme comme le marché du travail et les unions ouvrières, la carrière des relations industrielles et publie in-extenso les programmes d'après-guerre du mouvement ouvrier canadien.

Si on met de côté l'ouvrage de M. H. A. Logan, publié en langue anglaise en 1928: The History of Trade-Union Organization in Canada, qui est aujourd'hui démodé à cause de l'essor considérable qu'a pris le mouvement ouvrier surtout depuis 1939 le volume de M. Després est le seul du genre. Il faut savoir gré à l'auteur d'avoir présenté en 200 pages, d'une lecture facile et accessible à tous, les éléments nécessaires à une bonne compréhension du mouvement ouvrier canadien. Car, il ne faut pas Poublier, le syndicalisme est implanté chez nous pour y demeurer et il est un facteur important dans la vie de la nation. Ce n'est pas par ce qu'en disent les quotidiens, qui ne recherchent que le sensationnel, quand ils ne sont pas à la solde d'intérêts financiers. qu'on apprendra à le connaître sur son vrai jour. Syndiqués, employeurs et profanes trouveront un grand profit à le lire.

Gérard DION

(1) Volume de 205 pages, Fides, Montréal 1946. On peut se procurer cet ouvrage au prix de $\$ 1.50$ en s'adressant au Département des cet ouvrage au prix de $\$ 1.50$ en s'adressant au
relations industrielles, Université Laval, Québec.

\section{NOS COLLABORATEURS :}

Dion, Gérard, L. Th., L.Ph., M.Sc.Soc., professeur à la Faculté des sciences sociales de Laval et secrétaire de son Département des relations industrielles.

Drolet, Arthur, membre de l'Association professionnelle des Industriels, vice-président de F.-X. Drolet, Limitée et directeur de l'Action Sociale Limitée, Québec.

Picard, Gérard, B. A., président général de la Confédération des Travailleurs Catholiques du Canada. 